\title{
Dengue fusion peptides in interaction with model membranes - a fluorescence study
}

Danilo da Silva Olivier ${ }^{1,2}{ }^{\circ}$, Graziely Ferreira Cespedes $^{3} \oplus$, Wallance Moreira Pazin ${ }^{1,4} \oplus$, Eduardo Maffud Cilli ${ }^{3}$, Amando Siuiti Ito ${ }^{1+}$ (6)

1. University of São Paulo, Faculty of Philosophy, Sciences and Letters of Ribeirão Preto, Ribeirão Preto, Brazil.

2. Universidade Federal do Tocantins, Araguaína Campus, Araguaína, Brazil.

3. São Paulo State University, Institute of Chemistry, Araraquara, Brazil.

4. São Paulo State University, School of Technology and Applied Sciences, Presidente Prudente, Brazil.

+Corresponding author: Amando Siuiti Ito, Phone: +55 11 3021-4574, Email address: amandosi@ffclrp.usp.br

\section{ARTICLE INFO}

Article history:

Received: July 01, 2020

Accepted: October 19, 2020

Published: April xx, 2021

\section{Keywords}

1. dengue virus peptide

2. lipid peptide interaction

3. fluorescence spectroscopy

4. vesicles diffusion coefficient

\begin{abstract}
Dengue fever is a widespread infectious disease caused by Dengue viruses and responsible for millions of cases per year. One of the key steps during the infection is the fusion between the cell membrane and the lipidic bilayer of the virus, done by the glycoprotein envelope. At the tip of the envelope there is a fusion peptide widely conserved among the four known virus serotypes. Here dengue fusion peptides were

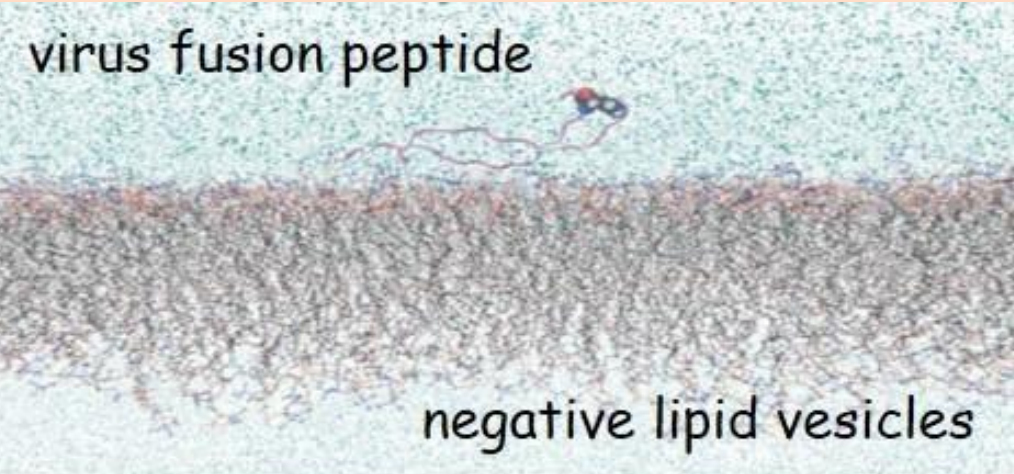
studied in buffer solution and interacting with model membranes using fluorescence techniques. Peptides have the tryptophan residue exposed to aqueous environment when in buffer, while is exposed to a hydrophobic environment when interacting with negatively charged vesicles, as shown by the blue shift of fluorescence emission and increase in the lifetime decay. Fluorescence anisotropy results confirm that the residue is in a more restrictive environment when interacting with vesicles. Finally, fluorescence correlation spectroscopy results support the importance of electrostatic interaction, showing that dengue peptide promotes a significant increase in diameter of negatively charged vesicles, compared to the absence of effect in the size of neutral vesicles.
\end{abstract}




\section{Introduction}

Dengue fever is an infectious disease spread in tropical and subtropical areas of the world, affecting around 2 billion people and causing as many as 100 million cases each year ${ }^{1,2}$. The disease is caused by the dengue virus, a member of the Flaviviridae family, a group of enveloped viruses containing a positivesense single-stranded RNA genome. The viral particle contains three structural proteins - capsid, membrane precursor and envelope glycoprotein protein (E) - and seven non-structural protein $\mathrm{s}^{3,4}$. As for other enveloped viruses, dengue virus infection depends on a step involving the fusion of the viral envelope with the cell membrane promoted by the envelope glycoprotein $\mathrm{E}^{5,6}$.
The glycoprotein E is a dimer complex with 495 amino acids in each subunit, which, under acidic conditions, undergoes a structural change, exposing the fusion peptide and forming a trimer ${ }^{7-10}$. The segment between residues 98 and 112, called fusion peptide, forms a loop in domain II of glycoprotein $\mathrm{E}$ and inserts into the cell membrane ${ }^{7,9,11,12}$ in the interaction step between the flavivirus and the host cell. The same sequence is present in the four dengue virus serotypes, which differ by the amino acid sequences flanking the fusion peptide. Dengue virus serotype II is associated with severe symptoms in humans, and the extended sequence 88-123, has been studied, trying to understand the process of fusion with membranes (Fig. 1).

\section{a)}

\section{KRFVC KHSMV DRGWG NGCGL FGKGG IVTCA MFTCKK 88 98 112

b)

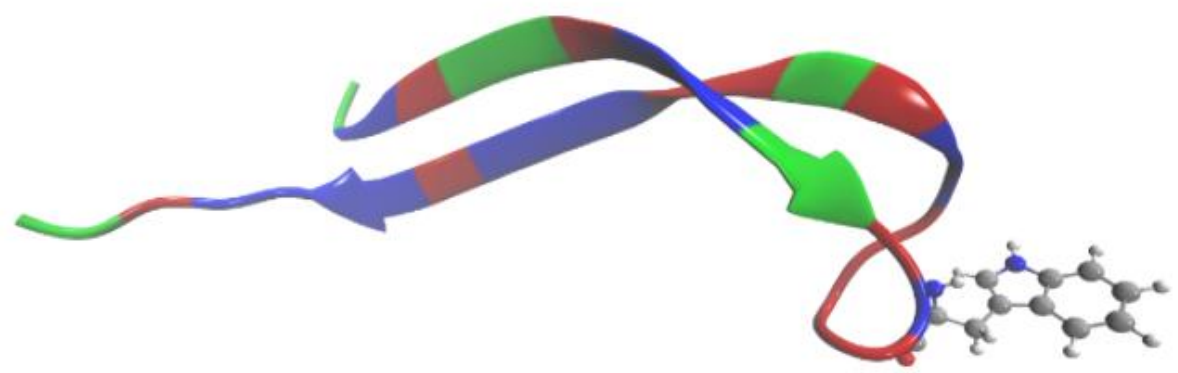

Figure 1. (a) Amino acid sequence of serotype II dengue virus fusion peptide (88-123); the sequence 98-112 is underlined, charged polar residues are indicated in green, hydrophobic residues are in blue and non-charged polar are in red. (b) Dengue peptide and tryptophan in new cartoon and CPK representation, respectively. Color code is the same as presented in (a). PDB ID:1OK $8^{3}$.

The peptide has a Trp residue in position 101 of the sequence, allowing its direct observation by fluorescence spectroscopy. In this study, we examined the fusion loop peptide and three other peptides from dengue virus serotype II: the long sequence $88-123$, the amino flank 88-111 and the carboxy flank 98-123. Due to the involvement in the process of virus fusion with cell membranes, we report here the analysis of the peptides in a buffer medium and interacting with model membranes using fluorescence spectroscopy techniques. As model membranes, we used small unilamellar vesicles (SUVs) of neutral lipid 1,2dimyristoyl-sn-glycero-3-phosphocoline (DMPC) and negative 1,2-dimyristoyl-sn-glycero-3-phosphoglycerol (DMPG). We also used large unilamellar vesicles (LUVs) of neutral lipid 1,2-dipalmitoyl-sn-glycero-3phosphocholine (DPPC) and negatively charged 1,2dipalmitoyl-sn-glycero-3-phosphoglycerol (DPPG).

\section{Experimental}

\subsection{Peptides synthesis}

Peptides were manually prepared using solid phase peptide synthesis by the 9-fluorenylmethoxylcarbonyl (Fmoc) procedure ${ }^{13}$. Ninhydrin test was used to monitor each coupling/deprotection steps. Amino acids were purchased in Nova Biochem Corp Synpep or Advanced Chem Tech (USA). PA solvents were acquired from LabSynth and Acros Organics (USA). Purification of products of synthesis was performed by preparative high performance liquid chromatography (HPLC) using C18 Phenomenex column. After the purification, peptides were characterized by analytical HPLC and mass spectrometry. 


\subsection{Preparation of model membranes}

The lipids DMPC, DMPG, DPPC and DMPG were purchased from Avanti Polar Lipids (Alabaster, AL, USA) as powder. The fluorescent probe 1,19dioctadecyl-3,3,39,39-tetramethylindocarbocyanine perchlorate (DiIC18) was from Molecular Probes (Eugene, OR, USA). All the reagents were used without further purification.

Small unilamellar vesicles films of DMPC and DMPG were prepared in standard test tubes by diluting the lipid in $20-30 \mu \mathrm{L}$ of chloroform, followed by evaporation of the solvent in a stream of $\mathrm{N}_{2}$. After this step, the tube is left under reduced pressure for $2 \mathrm{~h}$. Lipid dispersions were prepared by adding citrate buffer up to final concentration equal to $1 \mathrm{mmol} \mathrm{L}^{-1}$ lipid in $2 \mathrm{~mL}$ citrate buffer $\left(10 \mathrm{mmol} \mathrm{L}^{-1}, \mathrm{pH} \mathrm{5.4}\right)$. After vortexing in temperature above the lipid gel-fluid transition, the content was transferred into a Falcon with volume of $50 \mathrm{~mL}$.

The SUVs were prepared by sonication of the lipid dispersion using the Vibracell VC-600 sonicator, with $1 / 2$ in $(13 \mathrm{~mm})$ probe tip. The amplitude used during the sonication was set to $37 \%$. To measure the particle size, we used the Beckman coulter - N5 submicron particle size analyzer. The analysis was made by counts to obtain the percentage distribution and size of the particles.

Large unilamellar vesicles were prepared from stock solutions of DPPC and DPPG in chloroform at $40 \mathrm{mmol} \mathrm{L} \mathrm{L}^{-1}$. Dry films emerged by evaporating the solvent under a $\mathrm{N}_{2}$ flow and eliminating the remaining traces of organic solvent under reduced pressure for at least $3 \mathrm{~h}$. Multilamellar vesicles (MLVs) were prepared by adding Milli-Q water onto the films and vortexing them at $45{ }^{\circ} \mathrm{C}$ for $2 \mathrm{~min}$ above the phase transition temperature of DPPC. Subsequent extrusion afforded the LUVs, as described elsewhere ${ }^{14,15}$. The MLVs suspension was passed through a polycarbonate membrane with pores measuring $0.1 \mu \mathrm{m}$ (Whatman, Sigma Aldrich) at least 21 times.

\subsection{Optical absorption and fluorescence apparatus}

An Amersham Ultrospec 2100 pro spectrophotometer was used to obtain optical absorption spectra. Steady-state fluorescence measurements were made in a Hitachi F-7000 spectrofluorometer; internal calibration was performed to correct the emission spectra intensity, and polarizer filters were employed for anisotropy experiments.
Fluorescence intensity decays, and time-resolved anisotropy measurements were recorded on a picosecond laser system operating in the timecorrelated single-photon counting mode. The excitation source was a mode-locked Ti:sapphire laser (Tsunami 3950, pumped by Milennia X, Spectra Physics), which produced laser pulses with 5.0-ps Full Width at Half Maximum (FWHM). The pulse repetition rate was adjusted to $8.0 \mathrm{MHz}$ using the 3980 Spectra Physics pulse picker. The laser wavelength was selected with a third harmonic generator (BBO crystal, GWN-23PL Spectra Physics), yielding $295 \mathrm{~nm}$ excitation pulses that were directed to an L-format Edinburgh FL900 spectrometer with a monochromator in the emission channel. Single photons were detected by a cooled Hamamatsu R3809U microchannel plate photomultiplier, and the instrument response function was $~ 100$ ps. In anisotropy experiments, a SoleilBabinet compensator and a Glann-Taylor polarizer were used in the excitation and emission beam, respectively. Data were analyzed by using a commercial software (Edinburgh Instruments) based on nonlinear least squares method. The quality of the fit was judged by the reduced $\chi^{2}$ values and the residuals distribution.

\subsection{Fluorescence correlation spectroscopy}

Fluorescence correlation spectroscopy (FCS) experiments were performed in the PicoQuant MicroTime 200 instrument, which combined confocal optics (Olympus IX-71 inverted microscope) with pulsed excitation and time resolved data acquisition along with a highly sensitive avalanche photodiode detector. A pulsed diode laser operating at an excitation wavelength of $530 \mathrm{~nm}$ was employed, and detection was accomplished using a bandpass filter to collect light with a wavelength above $550 \mathrm{~nm}$ to remove scattered light. The samples were examined in a microscope slide, where a very small fluorescent volume was selected, and fluctuations in emission were collected. Using the software provided by PicoQuant, FCS measurements allowed determination of the autocorrelation function $G(t)$ for the fluorescence emission.

\section{Results and discussion}

\subsection{Peptides synthesis}

The sequence of the peptides obtained in the synthesis and used in this work are presented in Tab. 1. 
The peptides contain serine residues in substitution to cysteine in position 92, 105, 116 and 121. The cysteine residues were replaced by serine residues to avoid dimerization. In previous study, it was verified that this modification did not promote difference in fusion activity $^{16}$.

Table 1. Peptides sequence.

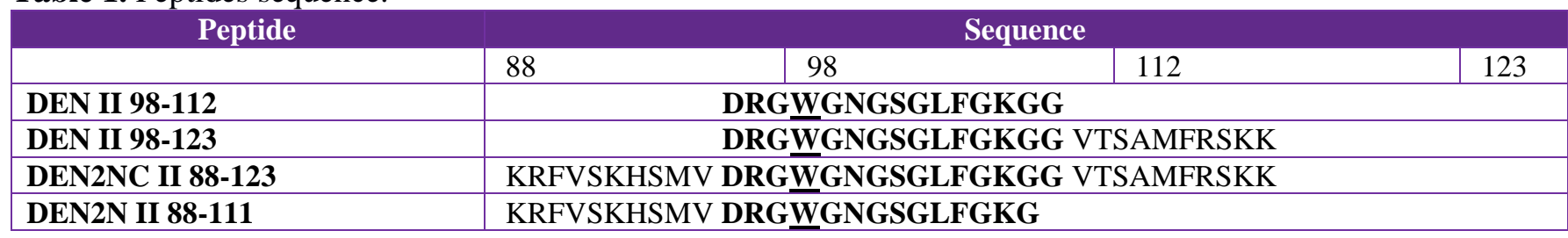

\subsection{Fluorescence of peptides in buffer}

\subsubsection{Emission spectra}

The peptides in citrate buffer $(10 \mathrm{mM}, \mathrm{pH} 5.4)$ solution presented absorption centered in $280 \mathrm{~nm}$ due to the presence of tryptophan residue. Under excitation at $290 \mathrm{~nm}$, the maximum of fluorescence emission was observed at $358 \mathrm{~nm}$ for DEN II 98-112 and DEN II 98-123 peptides and at $356 \mathrm{~nm}$ for DEN II 88-111 and DEN II $88-123$ peptides. The fluorescence spectra are typical of emission from tryptophan immersed in a polar environment (Fig. 2). Thus, the spectra observed for all peptides in citrate buffer solution indicate that the tryptophan residue in DEN II is exposed to the aqueous environment, independent of the sequences flanking the 98-112 fusion loop peptide.

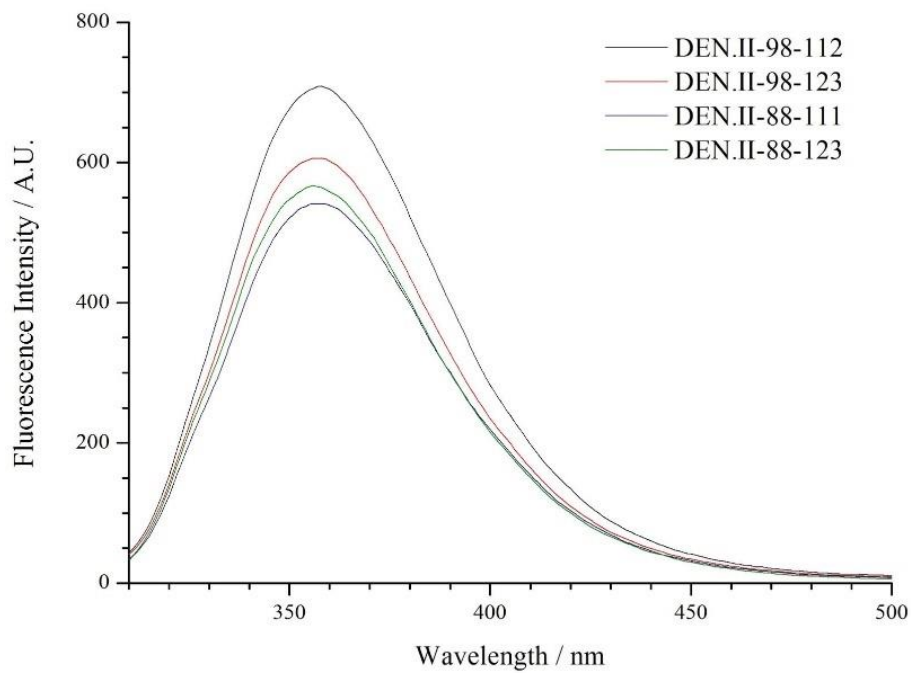

Figure 2. Fluorescence emission of dengue peptides in citrate buffer $\mathrm{pH}$ 5.4. Peptide concentration $15 \mu \mathrm{mol} \mathrm{L}{ }^{-1}$, excitation $290 \mathrm{~nm}$.

\subsubsection{Time-resolved emission}

Time-intensity $I(t)$ decays of all peptides measured at $350 \mathrm{~nm}$ were complex, and the experimental data were fitted to multi-exponential curves (Eq. 1):

$I(t)=I_{0} \sum_{i} \alpha_{i} e^{\frac{-t}{\tau_{i}}}$

where $I_{o}$ is the initial intensity, and $\alpha_{i}$ and $\tau_{i}$ are the normalized pre-exponential factor and the lifetime of component $i$ of the intensity decay, respectively. The best fit of intensity decay profiles of peptides in citrate buffer $\mathrm{pH} 5.4$ was obtained using three exponential functions, as usually found in peptides containing tryptophan (Fig. 3).

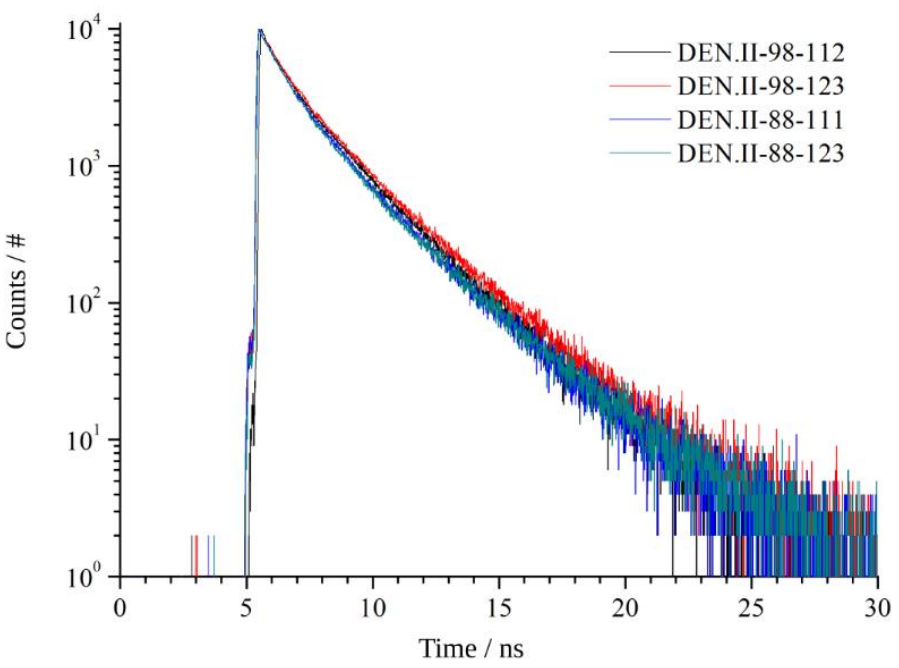

Figure 3. Time-resolved fluorescence decay for dengue II fusion peptides in citrate buffer $\mathrm{pH}$ 5.4. Excitation $290 \mathrm{~nm}$, temperature $45^{\circ} \mathrm{C}$.

The occurrence of three lifetimes in the decay of peptides was attributed to the presence of different rotational conformers of the indole ring around the $C_{\alpha^{-}}$ $C_{\beta}$ bond of the alanyl side chain: in the $g^{+}$(gauche plus) rotamers of $\operatorname{Trp}$ in the peptide chain, electron 
transfer processes predominate over fluorescence emission $^{17}$, leading to sub-nanosecond lifetime component. In the fitted curve for DEN II peptides, the value of the long lifetime component was around 3.0 $\mathrm{ns}$, and the intermediate component was circa $1.5 \mathrm{~ns}$; the sub-nanosecond component around $0.3 \mathrm{~ns}$ was necessary to fit the experimental decay (Tab. 2). The values are within those usually found in peptides, where the Trp residue is exposed to aqueous solvent ${ }^{18-21}$.

Table 2. Time-resolved data from fit of intensity decay to three exponential curves: lifetime components $\left(\tau_{\mathrm{i}}\right)$ and normalized pre-exponential factors $\left(\alpha_{\mathrm{i}}\right)$. Peptides in citrate buffer solution, $\mathrm{pH}$ 5.4. Concentration $15 \mu \mathrm{mol} \mathrm{L}^{-1}$ excitation $290 \mathrm{~nm}$, emission at $350 \mathrm{~nm}$. Temperature $45^{\circ} \mathrm{C}$.

\begin{tabular}{|lc|c|c|c|c|c|c|}
\hline \multirow{2}{*}{ Peptide } & \multicolumn{3}{c}{ Lifetime } & \multicolumn{3}{c|}{ Norm. pre-exponential } & \multicolumn{2}{c|}{ Average } \\
& $\tau_{1} / \mathrm{ns}$ & $\tau_{2} / \mathrm{ns}$ & $\tau_{3} / \mathrm{ns}$ & $\alpha_{1}$ & $\alpha_{2}$ & $\alpha_{3}$ & $<\tau>/ \mathrm{ns}$ \\
\hline \hline DEN II 98-112 & 2.88 & 1.47 & 0.25 & 0.15 & 0.49 & 0.36 & 1.87 \\
\hline DEN II 98-123 & 3.23 & 1.56 & 0.39 & 0.15 & 0.58 & 0.27 & 2.02 \\
\hline DEN II 88-111 & 3.62 & 1.53 & 0.26 & 0.05 & 0.63 & 0.32 & 1.75 \\
\hline DEN II 88-123 & 3.77 & 1.45 & 0.28 & 0.06 & 0.64 & 0.30 & 1.79 \\
\hline
\end{tabular}

The normalized pre-exponential factor for the short component was around 0.30 for all peptides. However, we can notice that the long lifetime component of the peptides with the amino flanking sequence (88-111 and 88-123) presents lower contribution to the decay (normalized pre-exponential around 0.05) compared to the fusion loop peptide (98-112) and the peptide with carboxy flanking sequence (98-123).

From the definition of mean values, the average lifetime $\langle\tau\rangle$ was calculated from individual lifetimes and corresponding pre-exponential factors according to Eq. 2:

$\langle\tau\rangle=\frac{\sum_{i} \alpha_{i} \tau_{i}^{2}}{\sum_{i} \alpha_{i} \tau_{i}}$

It is observed that smaller values of average lifetime correspond to the amino flanking peptides. The interaction of Trp with charged residues Arg, Lys and His may contribute to nonradiative de-excitation rate and subsequent decrease in fluorescent emission lifetime.

\subsubsection{Quenching of fluorescence emission}

The steady-state fluorescence emission intensity of peptides tryptophan decreased upon the addition of acrylamide into the solution. The decrease in intensity with increase in the concentration of quencher can be described by the Stern-Volmer equation (Eq. 3):

$\frac{F_{o}}{F}=1+K_{S V}[Q]$

where $F_{o}$ is the intensity in the solution without quencher, $F$ is the intensity in the presence of quencher at concentration $[Q]$, and $K_{S V}$ is the proportionality constant known as Stern-Volmer constant.
From time-resolved measurements, an equivalent equation can be written as Eq. 4.

$\frac{\tau_{o}}{\tau}=1+K_{d i n}[Q]$

where $\tau_{o}$ is the lifetime of the fluorescent molecule in the absence of quencher and $\tau$ is the lifetime in the presence of quencher at concentration $[Q]$, and $K_{\text {din }}$ is the proportionality constant known as dynamic constant for quenching.

Quenching of peptides emission was examined through the Stern-Volmer plot (Fig. 4). Both parameters $\mathrm{F}_{\mathrm{o}} / \mathrm{F}$ and $\tau_{\mathrm{o}} / \tau$ increased linearly with the quencher concentration and the Stern-Volmer constants $\left(K_{S V}\right)$ and the dynamic constant $\left(K_{d i n}\right)$, obtained from the respective plots, present good agreement (Tab. 3), demonstrating the collisional nature of quenching without the formation of nonfluorescent species.

a)

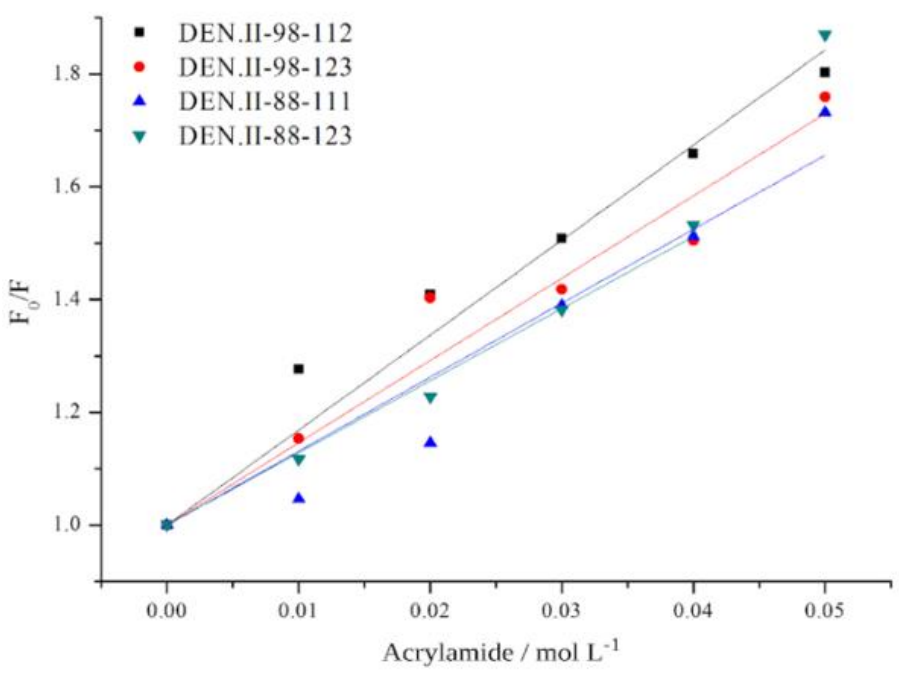


b)

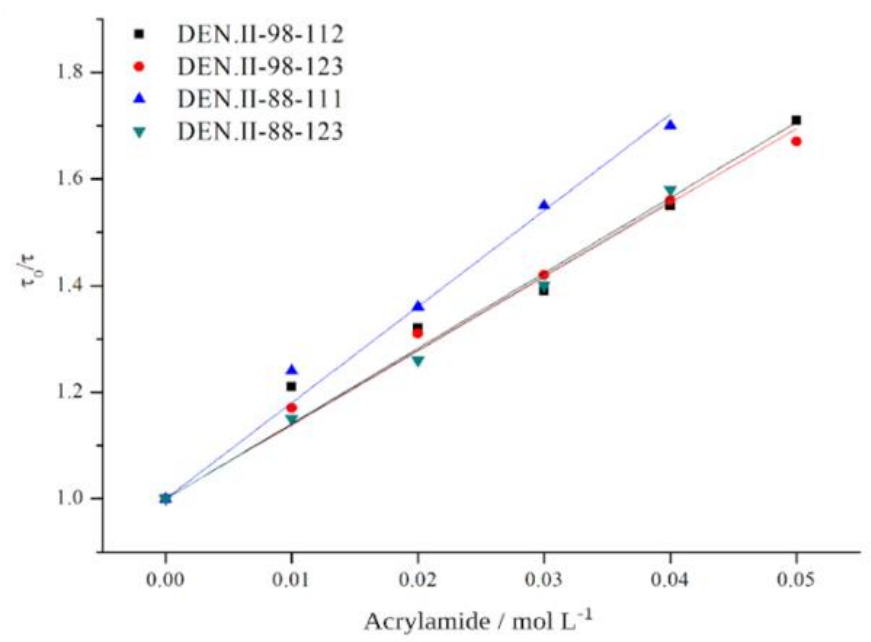

Figure 4. Quenching, by acrylamide, of tryptophan fluorescence in dengue II peptides. (a) Stern-Volmer plot using steady-state fluorescence emission. (b) Stern-Volmer plot from time-resolved data. Straight lines are best fits of experimental data to Stern-Volmer equation.

Results indicated that Trp residues in the peptides are exposed to the solvent, allowing the interaction with the quencher molecules. The quenching results from collisions between acrylamide molecules and tryptophan residues in the peptides are a process dependent on the diffusion of the interacting species in the medium. The collisional constant $K_{q}$ is proportional to the diffusion coefficients of acrylamide and peptides and is determined with Eq. 5.

$K_{q}=\frac{K_{\text {din }}}{\tau}$

In order to calculate the constant, we used the results of average lifetimes obtained according to Eq. 2. The values of collisional constant calculated for all the peptides (Tab. 3) are comparable to the results of observations made in antimicrobial and melanotropic peptides ${ }^{21,22}$ and are consistent with the dimensions of the molecules involved in the process.

The collisional constant is proportional to the sum of diffusion coefficients of fluorophore and quencher. We must consider that, in our experiments, the fluorophore is the tryptophan residue bound to the peptide chain, and there is another component in the process, related to the accessibility of the quencher to the indol group of tryptophan. Thus, the slightly higher value of $K_{q}$ observed for 88-111 peptide indicates an increased accessibility of the indol group to the quencher, suggesting that the tryptophan in the peptide has a high degree of exposure to the solvent.

Table 3. Steady state $\left(K_{s v}\right)$ and time-resolved $\left(K_{d i n}\right)$ constants for quenching of tryptophan emission by acrylamide. The collisional constants $\left(K_{q}\right)$ were calculated from Eq. 5.

\begin{tabular}{|l|c|c|c|} 
Peptide & $\begin{array}{c}\boldsymbol{K}_{\text {sv }} / \mathrm{L} \\
\mathrm{mol}^{-1}\end{array}$ & $\begin{array}{c}\boldsymbol{K}_{\text {din }} / \mathrm{L} \\
\mathrm{mol}^{-1}\end{array}$ & $\begin{array}{c}\boldsymbol{K}_{q} / \mathbf{1 0}^{\ominus} \mathrm{L} \\
\mathrm{mol}^{-1} \mathbf{s}^{-1}\end{array}$ \\
\hline DEN II 98-112 & 16.9 & 14.1 & 7.6 \\
\hline DEN II 98-123 & 14.6 & 13.9 & 6.9 \\
\hline DEN II 88-111 & 13.1 & 18.0 & 10.1 \\
\hline DEN II 88-123 & 12.8 & 14.0 & 7.7 \\
\hline
\end{tabular}

\subsubsection{Fluorescence anisotropy}

Values of steady-state fluorescence anisotropy $\left(A_{S S}\right)$ of the peptides measured in citrate buffer solution $\mathrm{pH}$ 5.4, around 0.020 (Tab. 4), are comparable to those observed in peptides with similar number of residues, like melanocyte stimulating hormone $(\mathrm{MSH})$ and adrenocorticotropin hormone fragment (ACTH 1$24)^{20,22}$. As the fluorescence anisotropy originates from the rotational movement of the fluorophore, the relatively low values of anisotropy reflect the rotational freedom of the peptides in aqueous medium. Slightly higher values were observed for the sequence 88-123 of the larger peptide.

Table 4. Steady-state anisotropy $\left(A_{S S}\right)$ and time-resolved components of anisotropy decay: rotational correlation time $\left(\varphi_{i}\right)$ and time-zero anisotropy $\left(\beta_{i}\right)$ of component i. Peptides in citrate buffer, $\mathrm{pH} 5.4$, concentration $15 \mu \mathrm{mol} \mathrm{L}^{-1}$, excitation $290 \mathrm{~nm}$, emission set at $350 \mathrm{~nm}$.

\begin{tabular}{|l|c|c|c|c|c|c|c|c|}
\hline Peptide & $\boldsymbol{A s s s}_{\text {ss }}$ & $\boldsymbol{\varphi}_{1} / \mathbf{n s}$ & $\boldsymbol{\varphi}_{2} / \mathbf{n s}$ & $\boldsymbol{\beta}_{1}$ & $\boldsymbol{\beta}_{2}$ & $\boldsymbol{r}_{\infty}$ & $\boldsymbol{r}_{\boldsymbol{O}}$ & $\boldsymbol{r}_{\text {mean }}$ \\
\hline $98-11245^{\circ} \mathrm{C}$ & 0.017 & 0.03 & 0.40 & 0.21 & 0.08 & -0.006 & 0.27 & 0.023 \\
\hline $98-12345^{\circ} \mathrm{C}$ & 0.019 & - & - & - & - & - & & \\
\hline $88-11145^{\circ} \mathrm{C}$ & 0.018 & 0.06 & 0.56 & 0.19 & 0.08 & -0.012 & 0.25 & 0.019 \\
\hline $88-12345^{\circ} \mathrm{C}$ & 0.021 & 0.08 & 0.55 & 0.15 & 0.11 & -0.014 & 0.25 & 0.027 \\
\hline $88-12325^{\circ} \mathrm{C}$ & 0.023 & 0.06 & 0.65 & 0.17 & 0.08 & 0.006 & 0.26 & 0.029 \\
\hline
\end{tabular}


Time-resolved data can offer elements for the analysis of steady-state results and the experimental anisotropy decays $r(t)$ were fitted to multi-exponential curves (Eq. 6):

$r(t)=\sum_{i} \beta_{i} e^{\frac{-t}{\varphi_{i}}}+r_{\infty}$

where $\beta_{i}$ and $\varphi_{i}$ are the time-zero anisotropy and rotational correlation time of component $i$ of the anisotropy decay, respectively, and $r_{\infty}$ stands for the residual anisotropy at long time $t$.

The fitted function for anisotropy decay relates to the theoretically derived expression ${ }^{23}$ (Eq. 7):

$r(t)=\left(r_{0}-r_{\infty}\right) \sum_{i} f_{i} e^{\frac{-t}{\varphi_{i}}}+r_{\infty}$

where $r_{o}$ is the initial anisotropy obtained from the experimental fit of anisotropy decay through (Eq. 8)

$r_{0}=\sum_{i} \beta_{i}+r_{\infty}$ and $f_{i}=\frac{\beta_{i}}{\sum_{i} \beta_{i}}$

From the usual definition of mean value, it is possible to calculate the mean anisotropy from the temporal dependence of intensity and anisotropy ${ }^{14}$, written from the fitted parameters as Eq. 9.

$r_{m}=\frac{\int_{0}^{\infty} r(t) I(t) d t}{\int_{0}^{\infty} I(t) d t}=r_{\infty}+\frac{1}{\sum_{i} \alpha_{i} \tau_{i}} \sum_{i, j} \frac{\alpha_{i} \beta_{j} \tau_{i} \varphi_{j}}{\left(\tau_{i}+\varphi_{j}\right)}$

Anisotropy decay profiles, as illustrated in Fig. 5 for DEN II 98-112, were best fitted to two rotational correlation times in the sub-nanosecond region (Tab. 4). For peptides, the short correlation time is ascribed to rotation of the Trp residue around its bonding to the peptide chain, and the longer correlation time corresponds to the whole peptide rotations ${ }^{21}$. The mean anisotropy, calculated from time-resolved data, has values comparable to the results of steady-state measurements. The calculated mean anisotropy contains contributions from fluorescence intensity decay and rotational movement of the fluorophore (Eq. 9). As the lifetime of Trp did not show large variation in the different peptides, the slightly higher value of anisotropy observed for the sequence 88-123 is consistent with the decrease in rotational diffusion of the longer peptide, associated to its larger dimension.

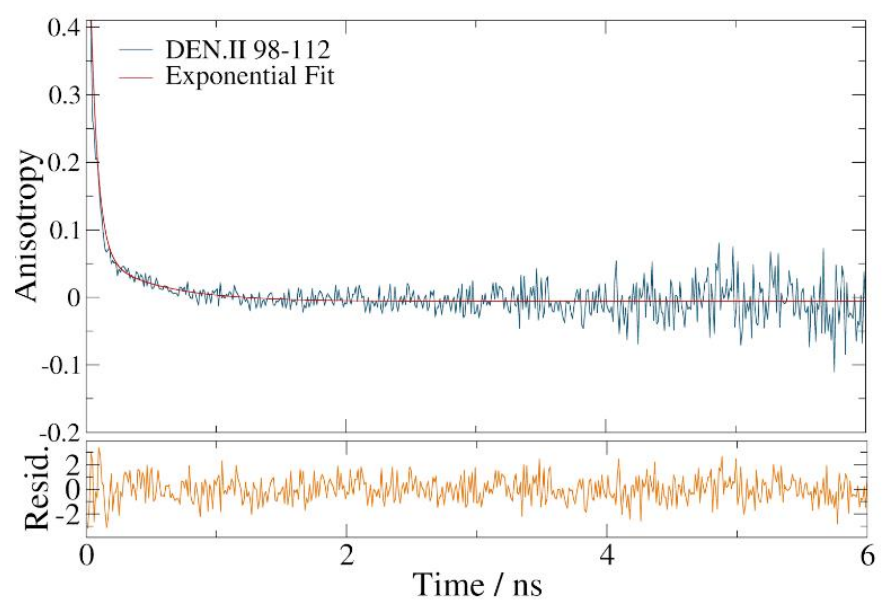

Figure 5. Fluorescence anisotropy decay for DEN.II 98-112 in buffer at $45^{\circ} \mathrm{C}$. Also shown best fit to a two exponential decay and graphic of residuals.

\subsection{Interaction with lipid vesicles}

The fluorescence of the long peptide $88-123$ was measured in the presence of SUVs and LUVs. SUVs of neutral (DMPC) and charged (DMPG) vesicles were prepared by sonication at $25{ }^{\circ} \mathrm{C}$. LUVs of neutral (DPPC) and charged (DPPG) lipids were prepared by extrusion at $45{ }^{\circ} \mathrm{C}$, above the vesicles thermal transition temperature.

In the presence of neutral PC vesicles, the spectral position of maximum emission did not change compared to the observations in aqueous buffer. In contrast, in the presence of negatively charged PG vesicles, we observed a $\sim 10 \mathrm{~nm}$ blue shift in the emission of tryptophan, accompanied by $\sim 25 \%$ increase in intensity, indicating the positioning of the fluorescent residue in a less polar environment in the acidic lipid vesicle. However, the extent of the spectral changes is not very large, indicating that the tryptophan is not inserted deep inside the bilayer. As the interaction occurs with negative lipids, there is also contribution of electrostatic effects, suggesting the positioning of the peptide in the region near to the negative charged phosphate groups of the lipids.

The results of the experiments of intensity time decay of the peptides emission in the presence of negatively charged DMPG and DPPG vesicles showed that the long lifetime component increased considerably, yielding to mean lifetime remarkably high compared to the values obtained in buffer (Tab. 5). The steady-state anisotropy changed markedly in the presence of vesicles, the more pronounced effect observed for the negatively charged LUVs or SUVs (Tab. 6). Furthermore, the long rotational correlation time attained values as high as 
3.2 ns in DMPG SUVs at $25{ }^{\circ} \mathrm{C}$ and $1.47 \mathrm{~ns}$ in DPPG LUVs at $45{ }^{\circ} \mathrm{C}$, showing that the rotational diffusion of the peptides greatly slowed down due to the interaction with negative vesicles. The increase in anisotropy and lifetimes is consistent with the insertion of the peptide in the interface region of the negatively charged vesicles $^{20,21}$, with consequent restriction to the rotational diffusion of tryptophan.

Table 5. Time-resolved data for emission of peptide 88-123 in the presence of lipid vesicles. Parameters obtained from fit of intensity decay to three exponential curves: lifetime components $\left(\tau_{i}\right)$ and normalized pre-exponential factors $\left(\alpha_{i}\right)$. Peptides in citrate buffer solution, pH 5.4. Concentration $20 \mu \mathrm{mol} \mathrm{L}{ }^{-1}$, excitation $290 \mathrm{~nm}$, emission set at $350 \mathrm{~nm}$. Temperature $45^{\circ} \mathrm{C}$.

\begin{tabular}{|l|c|c|c|c|c|c|c|}
\hline \multirow{2}{*}{ System } & \multicolumn{3}{c}{ Lifetime } & \multicolumn{3}{c|}{ Norm pre-exponential } & \multicolumn{1}{c|}{ Mean } \\
\hline Buffer $25^{\circ} \mathrm{C}$ & $\tau_{1} / \mathrm{ns}$ & $\tau_{2} / \mathrm{ns}$ & $\tau_{3} / \mathrm{ns}$ & $\alpha_{1}$ & $\alpha_{2}$ & $\alpha_{3}$ & $<\tau>/ \mathrm{ns}$ \\
\hline DMPC - SUV $25^{\circ} \mathrm{C}$ & 4.6 & 2.3 & 0.71 & 0.09 & 0.54 & 0.37 & 2.58 \\
\hline DMPG - SUV $25^{\circ} \mathrm{C}$ & 4.8 & 2.3 & 0.86 & 0.09 & 0.56 & 0.35 & 2.62 \\
\hline Buffer $45{ }^{\circ} \mathrm{C}$ & 7.1 & 3.2 & 1.20 & 0.05 & 0.58 & 0.36 & 3.40 \\
\hline DPPC - LUV $45^{\circ} \mathrm{C}$ & 3.8 & 1.5 & 0.28 & 0.06 & 0.64 & 0.30 & 1.81 \\
\hline DPPG - LUV $45^{\circ} \mathrm{C}$ & 8.0 & 1.7 & 0.14 & 0.05 & 0.50 & 0.45 & 3.54 \\
\hline
\end{tabular}

Table 6. Steady-state anisotropy $\left(A_{s s}\right)$ and time-resolved components of anisotropy decay: rotational correlation time $\left(\varphi_{i}\right)$ and time-zero anisotropy $\left(\beta_{i}\right)$ of component i. Peptides in citrate buffer, $\mathrm{pH} 5.4$, in medium containing suspension of lipid unilamellar vesicles. Peptide concentration $20 \mu \mathrm{mol} \mathrm{L} \mathrm{L}^{-1}$, lipid concentration $1 \mathrm{mmol} \mathrm{L}^{-1}$, excitation $290 \mathrm{~nm}$, emission at $350 \mathrm{~nm}$.

\begin{tabular}{|c|c|c|c|c|c|c|c|c|}
\hline System & $A_{\text {ss }}$ & $\varphi_{1} / \mathrm{ns}$ & $\varphi_{2} / \mathrm{ns}$ & $\beta_{1}$ & $\beta_{2}$ & $r_{\infty}$ & $\boldsymbol{r}_{o}$ & $r_{\text {mean }}$ \\
\hline Buffer $25^{\circ} \mathrm{C}$ & 0.023 & 0.06 & 0.65 & 0.17 & 0.08 & 0.002 & 0.260 & 0.029 \\
\hline DMPC/SUV $25^{\circ} \mathrm{C}$ & 0.034 & 0.06 & 0.67 & 0.14 & 0.07 & 0.010 & 0.253 & 0.035 \\
\hline DMPG/SUV $25^{\circ} \mathrm{C}$ & 0.083 & 0.07 & 3.20 & 0.21 & 0.02 & 0.020 & 0.250 & 0.067 \\
\hline Buffer $45^{\circ} \mathrm{C}$ & 0.021 & 0.08 & 0.55 & 0.15 & 0.11 & -0.014 & 0.246 & 0.024 \\
\hline DPPC/LUV $45^{\circ} \mathrm{C}$ & 0.042 & 0.04 & 0.45 & 0.18 & 0.10 & 0.018 & 0.296 & 0.042 \\
\hline DPPG/LUV $45^{\circ} \mathrm{C}$ & 0.065 & 0.06 & 1.47 & 0.16 & 0.07 & 0.015 & 0.246 & 0.049 \\
\hline
\end{tabular}

An interesting result was that increase in lifetime was not observed for the peptide in the presence of neutral SUVs of DMPC at $25{ }^{\circ} \mathrm{C}$. However, at the temperature of $45{ }^{\circ} \mathrm{C}$ the long lifetime component increased in the presence of neutral vesicles of DPPC, Thus, at high temperature the bilayer structure allows those neutral lipids to interact with the peptide with a significant increase in the calculated mean lifetime of Trp emission. The increase in temperature promotes thermal disorganization of the bilayer. At $25{ }^{\circ} \mathrm{C}$ the bilayers are in the gel phase and at $45{ }^{\circ} \mathrm{C}$, above the thermal phase transition temperature, the bilayers are in the liquid crystalline phase. In the high temperature the bilayer of the neutral lipids is less packed, making possible the positioning of the peptides so that the tryptophan residue can span regions in the bilayer with decreased polarity compared to the aqueous environment.

\subsection{Fluorescence correlation spectroscopy effects of the peptide on the size of vesicles}

In FCS measurements, software (SymPho Time 64) provided by PicoQuant, allowed determination of the auto-correlation function $G(t)$ for the fluorescence emission. The algorithm employed for data analysis, adequate to the low intensity emission from microscopic volumes, is based in a combination of time-correlated single-photon counting (TCSPC) technique and data acquisition following a specific time-tagged event, or TTTR (time-tagged timeresolved) mode ${ }^{24}$.

The time evolution of the fluctuations in intensity can be described by the autocorrelation function $G(t)$, which is dependent on the fluctuations in the concentration of the fluorophores. It was shown that, in the case of emission forming a single fluorescent species, the autocorrelation function relates to the diffusion of the fluorophore within a tiny sampling volume through Eq. 10. 
$G(t)=\frac{1}{N} \frac{1}{1+t / \tau_{D}}$

where $N^{\prime}$ and $\tau_{D}$ are the mean number of molecules and the diffusion time within the illuminated volume, respectively ${ }^{25}$.

The diffusion coefficient $D$ of the emitting subject is inversely proportional to $\tau_{D}$ and was determined from the experimental autocorrelation function. From $D$ values, the hydrodynamic radius $R_{H}$ of the fluorescent subject was calculated from the StokesEinstein equation (Eq. 11):

$R_{H}=\frac{k T}{6 \pi \eta D}$

where $k$ is the Boltzmann constant, and $\eta$ is the viscosity of the solution. In our experiments, the emitting species was the fluorescent probe DiIC $_{18}$. As the probe is bound to the vesicles, the diffusion coefficient and the hydrodynamic radius refer to the whole vesicles.

Fluorescence correlation spectroscopy experiments were performed in small unilamellar vesicles of DMPC and DMPG containing the fluorescent probe $\mathrm{DiIC}_{18}$ located in the surface vesicles, using a probe to lipid concentration below 1/100. From the measurements, we observed that the SUVs dimensions in buffer, calculated from the correlation curves, were comparable to those obtained from DLS (Tab. 7). After the addition of peptides, the FCS data for the neutral vesicles changed slightly, indicating the small extent of interaction that could not be observed through changes in the vesicle's diffusion. However, in the case of negative DMPC vesicles, the vesicles diffusion coefficient was significantly modified due to interaction with the peptides, resulting in a drastic increase in the diameter of the negative SUVs (Fig. 6).
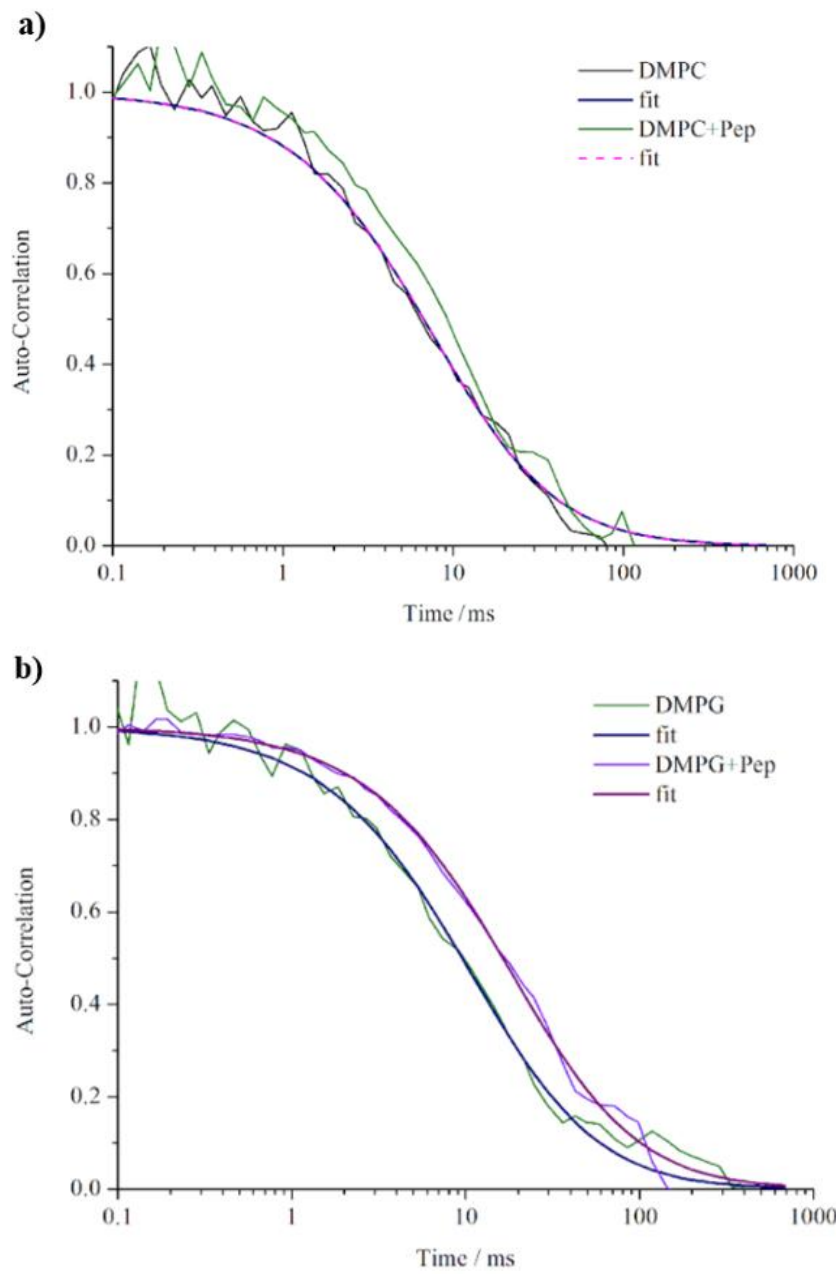

Figure 6. Autocorrelation function obtained from FCS experiments performed in small unilamellar vesicles of neutral DMPC and negative DMPG containing the fluorescent probe $\mathrm{DiIC}_{18}$. Experiments in citrate buffer $\mathrm{pH} 5.4$, at $45^{\circ} \mathrm{C}$, in the presence and in absence of DEN II 88-123 peptide.

Table 7. Diameter $(\Phi)$ of small unilamellar vesicles of DMPC and DMPG determined from DLS and FCS measurements in citrate buffer $\mathrm{pH} 5.4$, in the absence and in presence of DEN II 88-123 peptide. Also shown diffusion coefficient $(D)$ of the vesicles determined from FCS experiments.

\begin{tabular}{|c|c|c|c|}
\hline System & $\Phi / \mathrm{nm}$ - DLS & $D / \mu \mathrm{m}^{2} \mathrm{~s}^{-1}-\mathrm{FCS}$ & $\Phi / \mathrm{nm}-\mathrm{FCS}$ \\
\hline DMPC & 32.5 & - & - \\
\hline $\mathrm{DMPC}+\mathrm{DiIC}_{18}$ & 32.8 & 7.51 & 28.8 \\
\hline DMPC + DiIC $18+$ Peptide & nd & 8.18 & 26.4 \\
\hline DMPG & 46.0 & - & - \\
\hline $\mathrm{DMPG}+\mathrm{DiIC}_{18}$ & 47.5 & 8.43 & 25.6 \\
\hline DMPG + DiIC 18 + Peptide & nd & 3.24 & 67.0 \\
\hline
\end{tabular}

The FCS provides an experimental verification, independent of Trp fluorescence, revealing that the interaction between the DEN II 88-123 fusion peptide and the lipids vesicles has an electrostatic component, involving the charged residues of the peptide and the negatively charged head groups of DMPG vesicles. As a result of the interaction, the vesicles drastically 
changed shape and dimension, as revealed by the increase in the diffusion coefficient.

\section{Conclusions}

The fluorescence spectroscopy gives information about the local environment around the tryptophan residue in the dengue fusion peptides. The changes in fluorescence characteristics (emission spectrum, timeresolved intensity decay, quenching by acrylamide, steady-state and time-resolved anisotropy) are similar to observations performed in different peptides containing tryptophan, like melanocyte stimulating hormone, adrenocorticotropin hormone and antimicrobial peptides. Despite the local character of the information obtained from tryptophan fluorescence, it is possible to draw conclusions concerning the peptides. In buffer solution, intensities and lifetimes are smaller for the amino flanking peptides 88-111 and 88-123, due to the presence of positively charged amino acids Lys, Arg and His in the sequence that promotes nonradiative pathways to the de-excitation of the tryptophan excited state.

The dengue fusion peptides in the buffer have the tryptophan residue exposed to the aqueous environment, interacting with molecules dissolved in the medium. The extent of interaction with the quencher acrylamide depends on the size of the peptides, which affects their translational diffusion. The rotational diffusion was monitored by fluorescence anisotropy measurements and are resulted from the rotation of the Trp residue around its bonding to the peptide chain, combined with the rotation of the whole peptide. The model is consistent with the dependence of anisotropy on the size of the peptides.

The interaction with lipid vesicles was assessed by changes in spectral position, intensity and timeresolved parameters. Compatible with the role that the fusion loop peptide exerts in the process of entry in the cell, the fluorescence of tryptophan residue gives information about the insertion of the nonpolar sequence of fusion peptide into the nonpolar region of lipid vesicles. Noticeably, the effect is enhanced in the aggregates containing negatively charged phospholipids. The presence of positive residues in the amino flanking peptides indicates that the electrostatic and hydrophobic interactions are relevant to the process of interaction of serotype II dengue virus fusion peptide with the membrane. Results of FCS experiments using the membrane probe DiIC $_{18}$ reveal changes in the size of negative vesicles due to interaction with fusion peptides and are consistent with those obtained from tryptophan fluorescence.

\section{Acknowledgments}

EMC is a senior researcher of $\mathrm{CNPq}$ (grant 301975/2018-3). ASI is recipient of CNPq research grant (305771/2016-7). This study was financed in part by the Coordenação de Aperfeiçoamento de Pessoal de Nível Superior - Brasil (CAPES) - Finance Code 001.

\section{References}

[1] Bhatt, S., Gething, P. W., Brady, O. J., Messina, J. P., Farlow, A. W., Moyes, C. L., Drake, J. M., Brownstein, J. S., Hoen, A. G., Sankoh, O., Myers, M. F., George, D. B., Jaenisch, T., Wint, G. R. W., Simmons, C. P., Scott, T. W., Farrar, J. J., Hay, S. I., The global distribution and burden of dengue, Nature $496 \quad$ (2013) 504-507. https://doi.org/10.1038/nature12060.

[2] Barrows, N. J., Campos, R. K., Liao, K.-C., Prasanth, K. R., Soto-Acosta, R., Yeh, S.-C., Geraldine Schott-Lerner, G., Pompon, J., Sessions, O. M., Bradrick, S. S., Garcia-Blanco, M. A., Biochemistry and Molecular Biology of Flaviviruses, Chemical Reviews $118 \quad$ (8) (2018) 4448-4482. https://doi.org/10.1021/acs.chemrev.7b00719.

[3] Modis, Y., Ogata, S., Clements, D., Harrison, S. C., Structure of the dengue virus envelope protein after membrane fusion, Nature 427 (2004) 313-319. https://doi.org/10.1038/nature02165.

[4] Zonetti, L. F. C., Coutinho, M. C., Araujo, A. S. de, Molecular Aspects of the Dengue Virus Infection Process: A Review, Protein \& Peptide Letters 25 (8) (2018) 712-719. https://doi.org/10.2174/0929866525666180709115506.

[5] Smit, J. M., Moesker, B., Rodenhuis-Zybert, I., Wilschut, J., Flavivirus Cell Entry and Membrane Fusion, Viruses 3 (2) (2011) 160-171. https://doi.org/10.3390/v3020160.

[6] Rice, C. M., Linderbach, B. D., Theil, H., Flaviviridae: the viruses and their replication, In: Fields Virology 5th edition., Knipe, D. M., Howley PM, ed., Lippincott-Raven, Philadelphia, 2007, Ch. 33.

[7] Harrison, S. C., The $\mathrm{pH}$ sensor for flavivirus membrane fusion, Journal of Cell Biology 183 (2) (2008) 177-179. https://doi.org/10.1083/jcb.200809175.

[8] Hrobowski, Y. M., Garry, R. F., Michael, S. F., Peptide inhibitors of dengue virus and West Nile virus infectivity, Virology Journal 2 (2005) 49. https://doi.org/10.1186/1743422X-2-49.

[9] Seligman, S. J., Constancy and diversity in the flavivirus fusion peptide, Virology Journal 5 (2008) 27. https://doi.org/10.1186/1743-422X-5-27. 
[10] Stauffer, F., Melo, M. N., Carneiro, F. A., Sousa, F. J. R., Juliano, M. A., Juliano, L., Mohana-Borges, R., Poian, A. T. da, Castanho, M. A. R. B., Interaction between dengue virus fusion peptide and lipid bilayers depends on peptide clustering, Molecular Membrane Biology 25 (2) (2008) 128138. https://doi.org/10.1080/09687680701633091.

[11] Melo, M. N., Sousa, F. J. R., Carneiro, F. A., Castanho, M. A. R. B., Valente, A. P., Almeida, F. C. L., Poian, A. T. da, Mohana-Borges, R., Interaction of the Dengue Virus Fusion Peptide with Membranes Assessed by NMR: The Essential Role of the Envelope Protein Trp101 for Membrane Fusion, Journal of Molecular Biology 392 (3) (2009) 736-746. https://doi.org/10.1016/j.jmb.2009.07.035.

[12] Carpio, L. E., Villalaín, J., Identification of the phospholipid binding regions of the envelope $\mathrm{E}$ protein of flaviviruses by molecular dynamics, Journal of Biomolecular Structure and Dynamics 38 (17) (2020) 5136-5147. https://doi.org/10.1080/07391102.2019.1697368.

[13] Fields, G. B., Noble, R. L., Solid phase peptide synthesis utilizing 9-fluorenylmethoxycarbonyl amino acids, International Journal of Peptide and Protein Research 35 (3) (2009) 161-214. https://doi.org/10.1111/j.13993011.1990.tb00939.x.

[14] Ito, A. S., Rodrigues, A. P., Pazin, W. M., Barioni, M. B., Fluorescence depolarization analysis of thermal phase transition in DPPC and DMPG aqueous dispersions, Journal

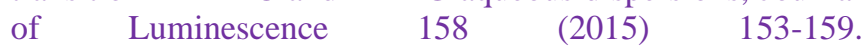
https://doi.org/10.1016/j.jlumin.2014.09.051.

[15] Ristori, S., Di Cola, E., Lunghi, C., Richichi, B., Nativi, C., Structural study of liposomes loaded with a GM3 lactone analogue for the targeting of tumor epitopes, Biochimica et Biophysica Acta (BBA) - Biomembranes 1788 (12) (2009) 2518-2525. https://doi.org/10.1016/j.bbamem.2009.10.005.

[16] Cespedes, G. F., Nobre, T. M., Oliveira Junior, O. N., Bong, D., Cilli, E. M., On the role of surrounding regions in the fusion peptide in dengue virus infection, Virology 557 (2021) 62-69. https://doi.org/10.1016/j.virol.2021.02.012.

[17] Goldman, C., Pascutti, P. G., Piquini, P., Ito, A. S., On the contribution of electron transfer reactions to the quenching of tryptophan fluorescence, The Journal of Chemical Physics 103 (24) (1995) 10614-10620. https://doi.org/10.1063/1.469846.

[18] Marquezin, C. A., Hirata, I. Y., Juliano, L., Ito, A. S., Tryptophan as a probe for acid-base equilibria in peptides, Peptide Science $71 \quad$ (5) (2003) 569-576. https://doi.org/10.1002/bip.10535.

[19] Fernandez, R. M., Vieira, R. F. F., Nakaie, C. R., Lamy, M. T., Ito, A. S., Acid-base titration of melanocortin peptides: Evidence of Trp rotational conformers interconversion, Biopolymers 80 (5) (2005) 643-650. https://doi.org/10.1002/bip.20210.

[20] Romani, A. P., Ito, A. S., Interaction of adrenocorticotropin peptides with microheterogeneous systems - A fluorescence study, Biophysical Chemistry 139 (2-3) (2009) 92-98. https://doi.org/10.1016/j.bpc.2008.10.009.

[21] Zanin, L. M. P., Alvares, D. dos S., Juliano, M. A., Pazin, W. M., Ito, A. S., Ruggiero Neto, J., Interaction of a synthetic antimicrobial peptide with model membrane by fluorescence spectroscopy, European Biophysics Journal 42 (11-12) (2013) 819-831. https://doi.org/10.1007/s00249-0130930-0.

[22] Souto, A. L. C. F., Ito, A. S., Tryptophan fluorescence studies of melanotropins in the amphiphile-water interface of reversed micelles, European Biophysics Journal 29 (1) (2000) 38-47. https://doi.org/10.1007/s002490050249.

[23] Kawato, S., Kinosita Junior, K., Ikegami, A., Effect of cholesterol on the molecular motion in the hydrocarbon region of lecithin bilayers studied by nanosecond fluorescence techniques, Biochemistry 17 (23) (1978) 50265031. https://doi.org/10.1021/bi00616a026.

[24] Wahl, M., Gregor, I., Patting, M., Enderlein, J., Fast calculation of fluorescence correlation data with asynchronous time-correlated single-photon counting, Optics $\begin{array}{lllllll}\text { Express } & 11 & \text { (26) } & \text { (2003) }\end{array}$ https://doi.org/10.1364/OE.11.003583.

[25] Krishevsky, O., Bonnet, G., Fluorescence correlation spectroscopy: the technique and its applications, Reports on Progress in Physics 65 (2) (2002) 251-297. https://doi.org/10.1088/0034-4885/65/2/203. 